\title{
Model-mapped random phase approximation to evaluate superconductivity in the fluctuation exchange approximation from first principles
}

\author{
Hirofumi Sakakibara ${ }^{1,2, *}$ and Takao Kotani ${ }^{1}$ \\ ${ }^{1}$ Department of Applied Mathematics and Physics, Tottori University, Tottori 680-8552, Japan \\ ${ }^{2}$ Computational Condensed Matter Physics Laboratory, RIKEN, Wako, Saitama 351-0198, Japan
}

(Received 10 December 2018; revised manuscript received 12 March 2019; published 23 May 2019)

\begin{abstract}
We have applied the model-mapped random phase approximation (RPA) [H. Sakakibara et al., J. Phys. Soc. Jpn. 86, 044714 (2017)] to the cuprate superconductors $\mathrm{La}_{2} \mathrm{CuO}_{4}$ and $\mathrm{HgBa}_{2} \mathrm{CuO}_{4}$, resulting in two-orbital Hubbard models. All the model parameters are determined based on first-principles calculations. For the model Hamiltonians, we perform fluctuation exchange calculation. Results explain relative height of $T_{c}$ observed in experiment for $\mathrm{La}_{2} \mathrm{CuO}_{4}$ and $\mathrm{HgBa}_{2} \mathrm{CuO}_{4}$. In addition, we give some analyses for the interaction terms in the model, especially comparisons with those of the constrained RPA.
\end{abstract}

DOI: 10.1103/PhysRevB.99.195141

\section{INTRODUCTION}

It is not so easy to treat strongly correlated electrons only by first-principles calculations. Thus we often use a procedure via a model Hamiltonian [1,2]; we determine a model Hamiltonian $\widehat{H}_{\mathrm{M}}$ from a first-principles calculation and then solve the model Hamiltonian. This is inevitable because firstprinciples calculations, which are mainly based on the density functional theory (DFT) in the local density approximation (LDA), are very limited to handle systems with correlated electrons. Widely used model Hamiltonians are the Hubbard ones, which consist of a one-body Hamiltonian $\widehat{H}_{\mathrm{M}}^{0}$ and the on-site interactions $\widehat{U}_{\mathrm{M}}$. To solve the Hubbard models, we can use a variety of methods [3-10] such as fluctuation exchange approximation (FLEX) [11].

To determine $\widehat{H}_{\mathrm{M}}$, we have formulated the model-mapped random phase approximation (mRPA) in Ref. [12] recently. In mRPA we use the standard procedure of the maximally localized Wannier function $[13,14]$ to determine $\widehat{H}_{\mathrm{M}}^{0}$. Here $\widehat{H}_{\mathrm{M}}^{0}$ is determined as a projection of the one-body Hamiltonian of first principles onto a model space, which is spanned by the Wannier functions. Then we determine $\widehat{U}_{M}$ so that the screened interaction of the model in the random phase approximation (RPA) agrees with that of the first principles. In this paper we consider on-site-only interaction in the model. Then we determine one-body double-counting term $\bar{U}_{\mathrm{M}}$. Finally we have $\widehat{H}_{\mathrm{M}}=\widehat{H}_{\mathrm{M}}^{0}+\widehat{U}_{\mathrm{M}}-\bar{U}_{\mathrm{M}}$.

mRPA can be taken as one of the improvements of cRPA $[15,16]$ in the sense to determine screened Coulomb interaction without screening effects from the model space. Until now, a variety of cRPA methods have been developed [17-36]. For example, Şaşıŏglu, Freidlich, and Blüegel $[23,32]$ developed a convenient cRPA method applicable to the case of entangled energy bands, while Miyake et al. [19] treated the case in a different manner. Nomura et al. showed a method to estimate the effective interaction for impurity problems in

*sakakibara.tottori.u@gmail.com
DMFT [25]. Casula et al. showed a method beyond the RPA to include the band renormalization effects [29].

In this paper, we apply mRPA to high- $T_{c}$ cuprate superconductors $\mathrm{La}_{2} \mathrm{CuO}_{4}\left(T_{c}=39 \mathrm{~K}\right.$ [37], denoted by La) and $\mathrm{HgBa}_{2} \mathrm{CuO}_{4}\left(T_{c}=98 \mathrm{~K}\right.$ [38], denoted by $\left.\mathrm{Hg}\right)$ to determine $\widehat{H}_{\mathrm{M}}$ of a two-orbital model [39-42]. After we determine $\widehat{H}_{\mathrm{M}}$, we perform FLEX calculations to investigate superconductivity. Our results are consistent with experiments. Since this mRPA+FLEX procedure can be performed without parameters by hand, we can claim that relative height of $T_{c}$ among materials is evaluated just from crystal structures. Thus, in principle, mRPA+FLEX can be used to find out a highest $T_{c}$ material among a lot of possible materials.

We like to emphasize the importance of the two-orbital model [39-42]. Although the Fermi surface of cuprates consists of the $d_{x^{2}-y^{2}}$ orbital mainly, Sakakibara et al. pointed out that hybridization of the $d_{x^{2}-y^{2}}$ orbital with the $d_{z^{2}}$ orbital [43-49] is very important. This can be represented by the two-orbital model. Sakakibara's FLEX calculation showed that the hybridization degrades spin-fluctuation-mediated superconductivity. This explains the difference of $T_{c}$ between $\mathrm{La}$ and $\mathrm{Hg}$ cuprates [39]. A recent photoemission experiment for La cuprate has captured significant orbital hybridization effects [50].

\section{METHOD}

Let us summarize the formulation of mRPA in Ref. [12]. First of all, we have to parametrize the interaction $\widehat{U}_{\mathrm{M}}$ of the model Hamiltonian so that $\widehat{U}_{\mathrm{M}}$ is specified by finite numbers of parameters. Figure 1 is a chart about how we determine $\widehat{H}_{\mathrm{M}}$. Step (1) is by first-principles calculations, and steps (2) and (3) are by model calculations. In this paper we will treat the on-site-only interaction of the two-orbital model specified by four parameters.

In step (1) of Fig. 1 we first perform a self-consistent calculation in first-principles method. Then we can obtain onebody Hamiltonian $\widehat{H}_{\mathrm{M}}^{0}$ in the standard procedure of maximally localized Wannier function [13,14]. In addition, we calculate 


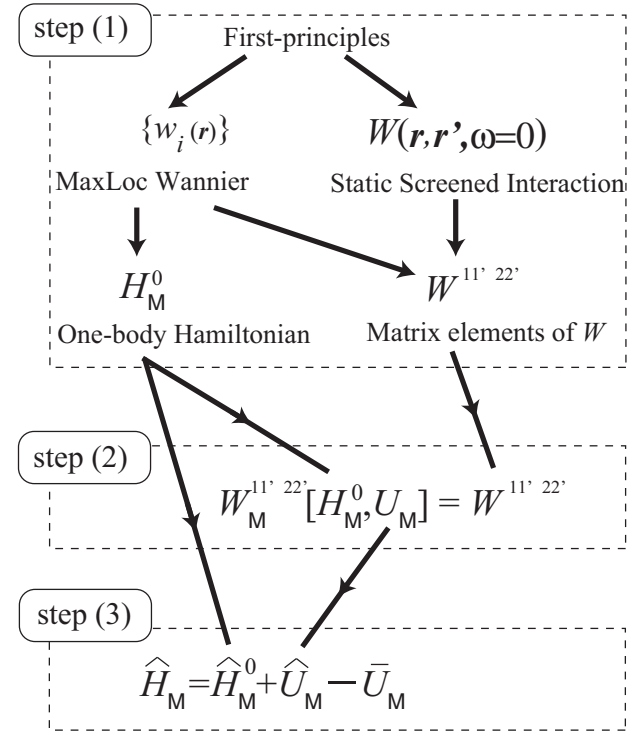

FIG. 1. How mRPA determines a model Hamiltonian $\widehat{H}_{\mathrm{M}}$. Note that quantities with subscript $\mathrm{M}$ are for the model Hamiltonian. At step (1), we obtain one-body Hamiltonian $H_{\mathrm{M}}^{0}$ and RPA screened Coulomb interaction $W^{11^{\prime} 22^{\prime}}$ in a first-principles calculation. At step (2), we obtain effective interaction $U_{\mathrm{M}}$ in the model, where we require $W_{\mathrm{M}}^{11^{\prime} 22^{\prime}}$ should be the same as $W^{11^{\prime} 22^{\prime}}$. At step (3), we determine $\bar{U}_{\mathrm{M}}$, which is to remove the double counting in the one-body term.

static screened Coulomb interaction $W\left(\mathbf{r}, \mathbf{r}^{\prime}, \omega=0\right)$ in RPA. Hereafter we omit $\omega=0$ since we treat only the static case in this paper. Then we calculate matrix elements $W^{11^{\prime 2} 22^{\prime}}$ of the matrix $W$, defined as

$$
\begin{aligned}
W^{11^{\prime} 22^{\prime}} & =\left(11^{\prime}|W| 22^{\prime}\right) \\
& =\int d^{3} \mathbf{r} d^{3} \mathbf{r}^{\prime} w_{1}^{*}(\mathbf{r}) w_{1^{\prime}}(\mathbf{r}) W\left(\mathbf{r}, \mathbf{r}^{\prime}\right) w_{2}^{*}\left(\mathbf{r}^{\prime}\right) w_{2^{\prime}}\left(\mathbf{r}^{\prime}\right),
\end{aligned}
$$

where $\left\{w_{1}(\mathbf{r})\right\}=\left\{w_{i_{1} \boldsymbol{R}_{\mathbf{1}}}(\mathbf{r})\right\}$ are the Wannier functions. $\boldsymbol{R}$ and $i$ denote a position of primitive cell and an orbital in each cell, respectively. The number of elements $W_{\mathrm{M}}^{11^{\prime 22} 2^{\prime}}$ is the same as the number of elements $U_{\mathrm{M}}^{11^{\prime} 22^{\prime}}$. Calculations are performed with ecalj package available from Git-hub [53].

In step (2) we determine $U_{\mathrm{M}}$, so that it satisfies

$$
W_{\mathrm{M}}^{11^{\prime} 22^{\prime}}\left[H_{\mathrm{M}}^{0}, U_{\mathrm{M}}\right]=W^{11^{\prime} 22^{\prime}},
$$

where a functional $W_{\mathrm{M}}^{11^{\prime 22}}\left[H_{\mathrm{M}}^{0}, U_{\mathrm{M}}\right]$ is a screened interaction in RPA calculated from $H_{\mathrm{M}}^{0}$ and $U_{\mathrm{M}}$. Here $H_{\mathrm{M}}^{0}$ denotes the matrix whose elements are $H_{\mathrm{M}}^{0,12} ; U_{\mathrm{M}}$ denotes the matrix whose elements are $U_{\mathrm{M}}^{11^{\prime} 22^{\prime}}$ as well. $\widehat{H}_{\mathrm{M}}^{0}$ is the second quantized operator made of the matrix $H_{\mathrm{M}}^{0}, \widehat{U}_{\mathrm{M}}$ as well. The functional is defined just in the model calculation; we do not treat quantities spatially dependent on $\mathbf{r}$. Equation (2) is a key assumption of mRPA; we require that the screened interaction in a model should be the same as those of theoretical correspondence in the first-principles calculation.

Let us detail the functional $W_{\mathrm{M}}^{11^{\prime} 22^{\prime}}\left[H_{\mathrm{M}}^{0}, U_{\mathrm{M}}\right]$. With noninteracting polarization function $P_{\mathrm{M}}\left[H_{\mathrm{M}}^{0}\right]$ of a model, we have effective interaction $W_{\mathrm{M}}$ in RPA as

$$
W_{\mathrm{M}}\left[H_{\mathrm{M}}^{0}, U_{\mathrm{M}}\right]=\frac{1}{1-U_{\mathrm{M}} P_{\mathrm{M}}\left[H_{\mathrm{M}}^{0}\right]} U_{\mathrm{M}} .
$$

Hereafter we omit $H_{\mathrm{M}}^{0}$ in $P_{\mathrm{M}}$ for simplicity. Here we only treat the nonmagnetic case. From Eq. (3) we have

$$
W_{\mathrm{M}}^{i_{1} i_{1} i_{2} i_{2} i_{2}^{\prime}}\left[H_{\mathrm{M}}^{0}, U_{\mathrm{M}}\right]=\frac{1}{N} \sum_{\boldsymbol{q}}\left[\frac{1}{1-U_{\mathrm{M}} P_{\mathrm{M}}(\boldsymbol{q})} U_{\mathrm{M}}\right]_{i_{1} i_{1^{\prime}} i_{2} i_{2^{\prime}}}
$$

for on-site interactions $U_{\mathrm{M}}$ and $W_{\mathrm{M}}$. Equation (4) is used in Eq. (2) so as to determine $U_{\mathrm{M}}$.

In step (3) we evaluate the one-body double counting term $\bar{U}_{\mathrm{M}}$ contained in the total model Hamiltonian $\widehat{H}_{\mathrm{M}}$. It is written as

$$
\widehat{H}_{\mathrm{M}}=\widehat{H}_{\mathrm{M}}^{0}+\widehat{U}_{\mathrm{M}}-\bar{U}_{\mathrm{M}} .
$$

To determine $\bar{U}_{\mathrm{M}}$, we require that the contribution from $\widehat{U}_{\mathrm{M}}$ and that from $\bar{U}_{\mathrm{M}}$ completely cancel when we treat $\widehat{U}_{\mathrm{M}}$ in a mean-field approximation. The mean-field approximation should theoretically correspond to the first-principle method from which we start. For example, if we use quasiparticle self-consistent $G W$ (QSGW) [54-56] as the first-principle method, we have to use QSGW to treat the model of Eq. (5). Then $\bar{U}_{\mathrm{M}}$ is made of the Hartree term and the static selfenergy term in the model. These terms cancel the effect of $\widehat{U}_{\mathrm{M}}$ when QSGW is applied too. In this case, we have reasonable theoretical correspondence between the first-principle calculation and model calculation. However, if we use LDA as the first-principle method, we have no corresponding meanfield approximation. Thus we cannot uniquely determine $\bar{U}_{\mathrm{M}}$. Instead of determining $\bar{U}_{\mathrm{M}}$, we use a practical method to avoid double counting in FLEX (see Sec. IV).

Let us recall the procedure of cRPA as a reference to mRPA. The effective interaction of cRPA $\left(U_{\mathrm{m}}\right)$ is determined based on the requirement

$$
\frac{1}{1-v P} v=\frac{1}{1-U_{\mathrm{m}} P_{\mathrm{m}}} U_{\mathrm{m}},
$$

where $v\left(\boldsymbol{r}, \boldsymbol{r}^{\prime}\right)$ is the bare Coulomb interaction, and $P_{\mathrm{m}}\left(\boldsymbol{r}, \boldsymbol{r}^{\prime}\right)$ is the polarization function within the model space spanned by the maximally localized Wannier functions. Equation (6) leads to

$$
U_{\mathrm{m}}=\frac{1}{1-v\left(P-P_{\mathrm{m}}\right)} v .
$$

Then we calculate the on-site matrix elements $U_{\mathrm{m}}^{122^{\prime} 1^{\prime}}=$ $\left(11^{\prime}\left|U_{\mathrm{m}}\right| 22^{\prime}\right)$.

Generally speaking, this cRPA procedure of Eq. (7) cannot be applicable to systems with entangled energy bands if the positive definiteness of $-\left(P-P_{\mathrm{m}}\right)$ in Eq. (7) is not satisfied. In fact, we have checked that $-\left(P-P_{\mathrm{m}}\right)$ do not satisfy the positive definiteness for $\mathrm{La}$ and $\mathrm{Hg}$. Thus we need to use a modified $P_{\mathrm{m}}$ satisfying the positive definiteness in a manner given by Şaşıoglu, Freidlich, and Blüegel $[23,32]$. In their method, such $P_{\mathrm{m}}$ is given in Eq. (60) in Ref. [32] as

$$
P_{\mathrm{m}}\left(\boldsymbol{r}, \boldsymbol{r}^{\prime}\right)=\sum_{i}^{\text {occ unocc }} \sum_{j} \frac{-2\left(c_{i} c_{j}\right)^{2} \phi_{i}(\mathbf{r}) \phi_{j}^{*}(\mathbf{r}) \phi_{j}\left(\mathbf{r}^{\prime}\right) \phi_{i}^{*}\left(\mathbf{r}^{\prime}\right)}{\epsilon_{j}-\epsilon_{i}},
$$

where $\phi_{i}$ is the eigenfunction. The probability factor $c_{i}$ is the norm for $\phi_{i}(\mathbf{r})$ projected into the model space spanned by the Wannier functions (see Eq. (58) in Ref. [32]). The composite index $i=(\mathbf{k}, n)$ is for the wave number $\mathbf{k}$ and the band index 
TABLE I. The interactions of mRPA $\left(U_{\mathrm{M}}\right)$ and cRPA $\left(U_{\mathrm{m}}\right)$ in a three-orbital model for $\mathrm{SrVO}_{3}$, where $d_{x y}, d_{y z}$, and $d_{z x}$ orbitals are considered. $U, U^{\prime}, J$ are the intraorbital, interorbital, and exchange interactions, respectively. The static screened interaction $W$ is also shown in the same manner as $U_{\mathrm{M}}$.

\begin{tabular}{lccc}
\hline \hline $\begin{array}{l}\mathrm{SrVO}_{3} \\
(\mathrm{eV})\end{array}$ & $W$ & $\begin{array}{c}\mathrm{mRPA} \\
U_{\mathrm{M}}\end{array}$ & $\begin{array}{c}\text { cRPA } \\
U_{\mathrm{m}}\end{array}$ \\
\hline$U$ & 0.852 & 2.82 & 3.12 \\
$U^{\prime}$ & 0.248 & 1.88 & 2.17 \\
$J$ & 0.290 & 0.442 & 0.448 \\
\hline \hline
\end{tabular}

$n$. Apparently, $0 \leqslant c_{\mathbf{k} n} \leqslant 1$ and $\sum_{n}\left(c_{\mathbf{k} n}\right)^{2}=1$ are satisfied for given $\mathbf{k}$. Thus $-\left(P-P_{\mathrm{m}}\right)$ is clearly positive definite because it is calculated just from the equation with $1-\left(c_{i} c_{j}\right)^{2}$ instead of $-\left(c_{i} c_{j}\right)^{2}$ in the numerator of Eq. (8).

As a check for our implementation of mRPA and cRPA, we show $U_{\mathrm{m}}$ and $U_{\mathrm{M}}$ for $\mathrm{SrVO}_{3}$ where three $3 d$ bands spanning model space are clearly separated from the other bands. In this case, we can expect that nonzero $c_{i}$ are not widely distributed among energy bands. Only $c_{i}$ for the three $3 d$ bands are almost unity, while others are almost zero. In this case, as shown in Table I, $U_{\mathrm{m}}$ is close to $U_{\mathrm{M}}: U$ of $U_{\mathrm{M}}, 2.82 \mathrm{eV}$, is only a little smaller than $U$ of $U_{\mathrm{m}}, 3.12 \mathrm{eV}$. This is reasonable since both mRPA and cRPA are to remove the screening effect related to the model space, although we treat only the on-site interactions in mRPA. The difference $2.82-3.12=-0.30 \mathrm{eV}$ may be mainly explained by the effect of off-site interactions. To check this, we apply mRPA using Eq. (9) of Ref. [12] including the interactions between all vanadium sites. In this case, the values obtained in MRPA should be in agreement with that of cRPA in principle. We find that $U$ of $U_{\mathrm{M}}$ becomes larger [57] to be $3.33 \mathrm{eV}$, slightly overshoots but becomes closer to $3.12 \mathrm{eV}$. Still the remaining difference 3.33-3.12= $0.21 \mathrm{eV}$ may be due to detailed differences of formalisms and numerical treatment.

\section{RESULT FOR EFFECTIVE INTERACTION}

Following the chart of Fig. 1, we apply mRPA to singlelayered cuprates, $\mathrm{La}$ and $\mathrm{Hg}$, to obtain the two-orbital Hubbard model [39], where we start from LDA calculations. We show their experimental crystal structures [51,52] in Fig. 2, together with their LDA band structures in Figs. 2(b) and 2(d),
TABLE II. The interactions of mRPA $\left(U_{\mathrm{M}}\right)$ and cRPA $\left(U_{\mathrm{m}}\right)$ for the experimentally observed crystal structure of $\mathrm{La}_{2} \mathrm{CuO}_{4}$ and $\mathrm{HgBa}_{2} \mathrm{CuO}_{4}$ [51,52]. The elements of $W$ are defined in the same manner as $U_{\mathrm{M}}$ (see text).

\begin{tabular}{lccc}
\hline \hline $\begin{array}{l}\mathrm{La}_{2} \mathrm{CuO}_{4} \\
(\mathrm{eV})\end{array}$ & $W$ & $\begin{array}{c}\mathrm{mRPA} \\
U_{\mathrm{M}}\end{array}$ & $\begin{array}{c}\mathrm{cRPA} \\
U_{\mathrm{m}}\end{array}$ \\
\hline$U^{x^{2}-y^{2}}$ & 0.747 & 2.76 & 3.14 \\
$U^{z^{2}}$ & 1.58 & 2.63 & 2.95 \\
$U^{\prime}$ & 0.370 & 1.64 & 2.01 \\
$U^{J}$ & 0.273 & 0.44 & 0.41 \\
$\mathrm{HgBa}_{2} \mathrm{CuO}_{4}$ & & $\mathrm{mRPA}$ & $\mathrm{cRPA}$ \\
$(\mathrm{eV})$ & $W$ & $U_{\mathrm{M}}$ & $U_{\mathrm{m}}$ \\
\hline$U^{x^{2}-y^{2}}$ & 0.820 & 2.99 & 2.14 \\
$U^{z^{2}}$ & 3.83 & 5.47 & 4.93 \\
$U^{\prime}$ & 0.724 & 2.62 & 1.92 \\
$U^{J}$ & 0.460 & 0.67 & 0.58 \\
\hline \hline
\end{tabular}

where we superpose the energy bands of the two-orbital models. In addition, we treat hypothetical cases varying apical oxygen height $h_{\mathrm{O}}$ in La [Figs. 2(a) and 2(c)] in order to clarify differences between MRPA and cRPA. Here $h_{\mathrm{O}}$ is defined as the distance shown in Fig. 2. The matrix $U_{\mathrm{M}}$ of the two-orbital model is represented as

$$
U_{\mathrm{M}}=\left(\begin{array}{cccc}
U^{x^{2}-y^{2}} & 0 & 0 & U^{\prime} \\
0 & U^{J^{\prime}} & U^{J} & 0 \\
0 & U^{J} & U^{J^{\prime}} & 0 \\
U^{\prime} & 0 & 0 & U^{z^{2}}
\end{array}\right),
$$

where the indices of the matrix $U_{\mathrm{M}}$ takes $d_{x^{2}-y^{2}} d_{x^{2}-y^{2}}$, $d_{x^{2}-y^{2}} d_{z^{2}}, d_{z^{2}} d_{x^{2}-y^{2}}$, and $d_{z^{2}} d_{z^{2}}$. Here $U^{\prime}$ are interorbital Coulomb interactions and $U^{J}=U^{J^{\prime}}$ are exchange interactions. Other interactions such as $W_{\mathrm{M}}$ are represented as well.

In Table II we show values of $U_{\mathrm{M}}$ for La and Hg [Figs. 2(b) and 2(d)], together with values of $W$ [58]. At first, let us compare $W$ for $\mathrm{La}$ and $\mathrm{Hg}$. We see a little difference on $W^{x^{2}-y^{2}}$ $(0.747 \mathrm{vs} 0.820 \mathrm{eV})$, while a larger difference on $W^{z^{2}}(1.58$ vs $3.83 \mathrm{eV}$ ). This is expected since $\mathrm{Hg}$ is more anisotropic than La, as indicated by the size of $h_{\mathrm{O}}$. From these $W$ and the band structure of the two-orbital model, we have obtained $U_{\mathrm{M}}$ shown in Table II. We see that ratios $U_{\mathrm{M}} / W$ are similar for $\mathrm{La}$ and $\mathrm{Hg}$, that is, $2.76 / 0.747 \sim 2.99 / 0.820$ for $W^{x^{2}-y^{2}}$, other

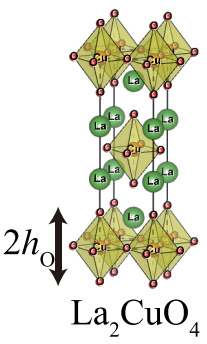

(a) $h_{\mathrm{O}}=2.30 \AA$

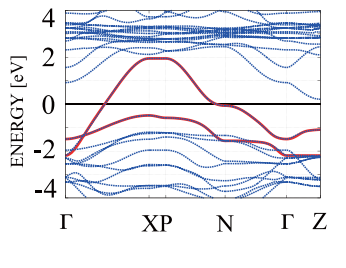

(b) $h_{\mathrm{O}}=2.41 \AA$

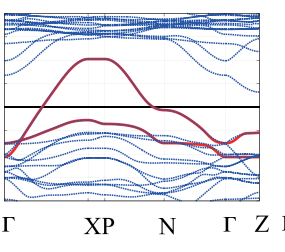

(c) $h_{\mathrm{O}}=2.50 \AA$

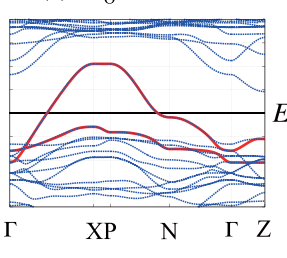

(d) $h_{\mathrm{o}}=2.78 \AA$

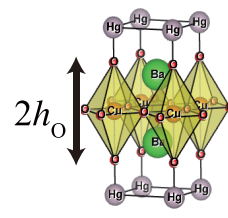

$\mathrm{HgBa}_{2} \mathrm{CuO}_{4}$

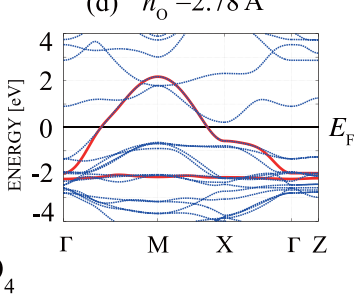

FIG. 2. Crystal structures and band structures of $\mathrm{La}_{2} \mathrm{CuO}_{4}$ (a)-(c) and $\mathrm{HgBa}_{2} \mathrm{CuO}_{4}$ (d). Blue dashed lines are for the LDA band structures; red solid lines are for the two-orbital models. The cases (a)-(c) are for varying the apical oxygen height $h_{\mathrm{O}}$. The cases (b) and (d) are with the experimental $h_{\mathrm{O}}[51,52]$. 
elements as well. This is consistent with the similarity of the band structure shown in Figs. 2(b) and 2(d).

We find that $U_{\mathrm{M}}^{x^{2}-y^{2}}$ is roughly estimated by

$$
U_{\mathrm{M}}^{x^{2}-y^{2}} \sim \frac{W^{x^{2}-y^{2}}}{1+W^{x^{2}-y^{2}} P_{\mathrm{M}}^{x^{2}-y^{2}}},
$$

where $P_{\mathrm{M}}^{x^{2}-y^{2}}$ is the diagonal elements of the Brillouin zone average of $P_{\mathrm{M}}(\boldsymbol{q})$. Equation (10) is derived from Eq. (4) by replacing $P_{\mathrm{M}}(\boldsymbol{q})$ with the average. Let us evaluate Eq. (10). Our calculation gives $P_{\mathrm{M}}^{x^{2}-y^{2}}=-0.97 \mathrm{eV}^{-1}$ for $\mathrm{La}$ and $-0.91 \mathrm{eV}^{-1}$ for $\mathrm{Hg}$. The little difference $-0.06=(-0.97)-$ $(-0.91) \mathrm{eV}^{-1}$ corresponds to the little difference of the band structures of the two-orbital models shown in Figs. 2(b) and 2(d). Together with the values of $W^{x^{2}-y^{2}}=0.747,0.820 \mathrm{eV}$ in Table II, Eq. (10) gives $U_{\mathrm{M}}^{x^{2}-y^{2}} \sim 2.71 \mathrm{eV}$ for La and $\sim 3.23 \mathrm{eV}$ for $\mathrm{Hg}$. These are roughly in agreements with $U_{\mathrm{M}}^{x^{2}-y^{2}}=2.76$ and $2.99 \mathrm{eV}$ in Table II. This analysis indicates that the difference of $U_{\mathrm{M}}^{x^{2}-y^{2}}$ between $\mathrm{La}$ and $\mathrm{Hg}$ is mainly due to the difference of $W^{x^{2}-y^{2}}$.

In Table II we also show cRPA values $U_{\mathrm{m}}$ for comparison. For La, Table II shows that $U_{\mathrm{m}}$ gives good agreement with $U_{\mathrm{M}}$, a little smaller as in the case of $\mathrm{SrVO}_{3}$ in Table I. On the other hand, we see large discrepancy for $\mathrm{Hg}: U_{\mathrm{m}}^{x^{2}-y^{2}}=2.14 \mathrm{eV}$ is much smaller than $U_{\mathrm{M}}^{x^{2}-y^{2}}=2.99 \mathrm{eV}$. This difference can be explained by Eq. (8) with factors $c_{i}$. In $\mathrm{Hg}$, we see a stronger $d-p$ hybridization in Fig. 2(d) than La; the position of $\mathrm{Cu}-$ $d_{x^{2}-y^{2}}$ band is pushed down to be in the middle of the oxygen bands. This means that nonzero $c_{i}$ are more distributed among the oxygen bands in the case of $\mathrm{Hg}$ than in the case of $\mathrm{La}$. This can be a reason to make the effective size of $P_{\mathrm{m}}$ smaller than $P_{\mathrm{M}}$ in the case of $\mathrm{Hg}$, resulting in the smaller $U_{\mathrm{m}}$.

To confirm the effect of hybridization, we calculate $U_{\mathrm{m}}$ and $U_{\mathrm{M}}$ by varying $h_{\mathrm{O}}$ for La. As discussed in Ref. [39], $h_{\mathrm{O}}$ is a key quantity to determine the critical temperatures of superconductors [59-64]. We can see $h_{\mathrm{O}}$ works as a control parameter of hybridization $[34,63,64]$. That is, as shown in Figs. 2(a)-2(c), higher $h_{\mathrm{O}}$ pushes down $\mathrm{Cu}-d_{x^{2}-y^{2}}$ levels more, resulting in larger hybridization with oxygen bands. Figure 2(d) for $\mathrm{Hg}$ can be taken as a case with highest $h_{\mathrm{O}}$.

In Fig. 3 we plot $U_{\mathrm{M}}$ and $U_{\mathrm{m}}$ together with $W$. Let us focus on Figs. 3(a) and 3(e). As a function of $h_{\mathrm{O}}, W^{x^{2}-y^{2}}$ is almost constant. In addition, the energy bands of the two-orbital model change little as shown in Figs. 2(a)-2(c). Thus it is reasonable that $U_{\mathrm{M}}^{x^{2}-y^{2}}$ changes little in Fig. 3(a), because of Eq. (10). On the other hand, $U_{\mathrm{m}}^{x^{2}-y^{2}}$ decreases rapidly when $h_{\mathrm{O}}$ becomes higher. This means that $P_{\mathrm{m}}$ becomes smaller for higher $h_{\mathrm{O}}$. As in the case of the $\mathrm{Hg}$ case, we think this is because of larger hybridization of $\mathrm{Cu}-d_{x^{2}-y^{2}}$ bands with oxygen bands.

Our mRPA and cRPA results are rather different. In Ref. [34] we treated a variety of layered cuprates, where we show that the effective interaction for $\mathrm{La}$ is larger than that for $\mathrm{Hg}$ as shown by $U_{\mathrm{m}}$ in Table II, based on the cRPA calculations. In addition, we showed the effective interactions are controlled by $h_{\mathrm{O}}$ as shown in $U_{\mathrm{m}}$ in Fig. 3. Even though we do not need to modify the overall conclusion in Ref. [34],
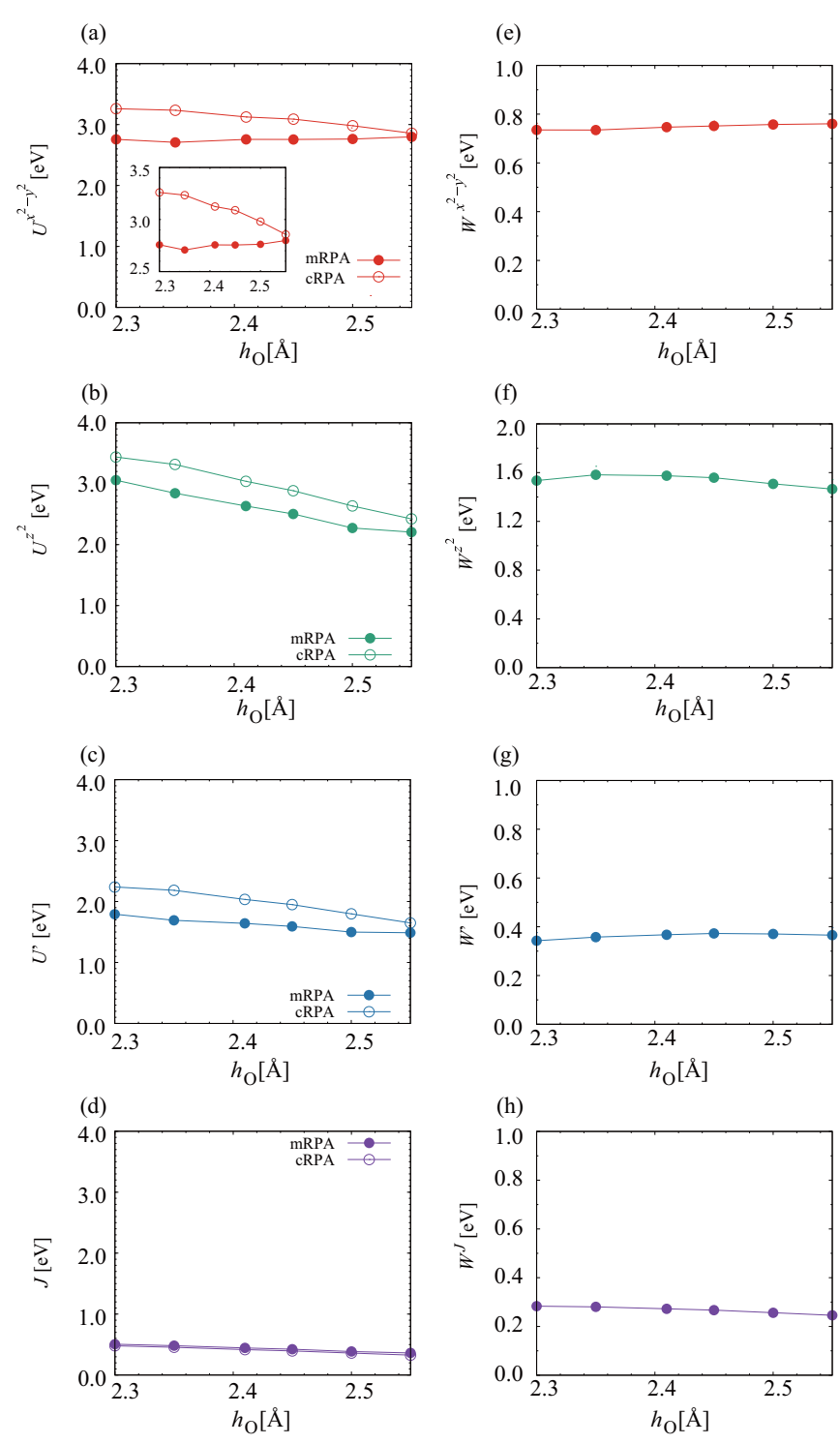

FIG. 3. The elements of $U_{\mathrm{M}}$ (mRPA), $U_{\mathrm{m}}$ (cRPA), and $W$ are plotted as a function of $h_{\mathrm{O}}$. Details of numerical settings are shown in the text. Note that $W_{\mathrm{M}}^{11^{\prime} 22^{\prime}}\left[U_{\mathrm{M}}\right]=W^{11^{\prime 222^{\prime}}}$ is satisfied at any values of $h_{\mathrm{O}}$. (a) and (e) indicate that $U^{x^{2}-y^{2}}$ for cRPA is affected by the $d-p$ hybridization (see text).

we should not take such effective interactions as suitable for Hubbard models. Along the logic of mRPA, we should use $U_{\mathrm{M}}$ instead of $U_{\mathrm{m}}$.

\section{FLEX CALCULATION FOR SUPERCONDUCTIVITY}

For the model Hamiltonian $\widehat{H}_{\mathrm{M}}$ obtained from mRPA, we perform two-orbital FLEX calculations to obtain dressed Green's functions $G_{i j}(k)$ [11,65-68]. Here $k=\left(\boldsymbol{k}, i \omega_{n}\right)$ is a composite index made of the wave vector $\boldsymbol{k}$ and the Matsubara frequency $i \omega_{n}$. The band index $i$ takes 1 or 2 . We calculate only the optimally doped case for $T_{c}$ (15\% doping). We take $32 \times 32 \times 4 k$ meshes and 1024 Matsubara frequencies.

Let us remind of step (3) in Fig. 1 to determine the counter one-body term $\bar{U}_{\mathrm{M}}$. Instead of LDA, let us consider QSGW case first. Theoretically, it is easier since QSGW is 
a method directly applicable even to a model Hamiltonian, where QSGW determines a mean-field one-body Hamiltonian for the model. We first determine $\widehat{H}_{\mathrm{M}}^{0}$ in QSGW by the firstprinciple QSGW calculation and the Wannier function method in step (1) of mRPA. Then we can determine $\widehat{U}_{\mathrm{M}}$ in step (2) of mRPA. In step (3) we apply the QSGW method to the model Hamiltonian $\widehat{H}_{\mathrm{M}}=\widehat{H}_{\mathrm{M}}^{0}+\widehat{U}_{\mathrm{M}}-\bar{U}_{\mathrm{M}}$, where yet an unknown term $\bar{U}_{\mathrm{M}}$ is included. Here $\bar{U}_{\mathrm{M}}$ is determined so that the QSGW applied to $H_{\mathrm{M}}$ do give the mean-field one-body Hamiltonian $\widehat{H}_{\mathrm{M}}^{0}$. That is, the effect of $\widehat{U}_{\mathrm{M}}$ to the one-body Hamiltonian is completely canceled by $\bar{U}_{\mathrm{M}}$.

When we start from LDA instead of QSGW, we have no unique way to determine $\bar{U}_{\mathrm{M}}$ since LDA cannot be applicable to the model Hamiltonian. Thus we need some assumption to follow the case of QSGW. Here we identify the static part of the self-energy $\Sigma(\boldsymbol{k}, 0)$ as $\bar{U}_{\mathrm{M}}$ [our definition of $\Sigma(\boldsymbol{k}, 0)$ here includes the Hartree term]. In other words, if we perform a static FLEX calculation only with $\Sigma(\boldsymbol{k}, 0)$, we reproduce the one-body Hamiltonian of LDA. This method is equivalent to Eq. (5) in Ref. [69]. We simply assume FLEX is not for the mean-field part, but for the $\omega$-dependent self-energy part.

Here we investigate superconductivity in the two-orbital model. By substituting $G_{i j}(k)$ into the linearized Eliashberg equation,

$$
\begin{aligned}
\lambda \Delta_{i j}(k)= & -\frac{T}{N} \sum_{q, m_{i}} V_{i m_{1} m_{4} j}(q) G_{m_{1} m_{2}}(k-q) \\
& \times \Delta_{m_{2} m_{3}}(k-q) G_{m_{4} m_{3}}(-k+q),
\end{aligned}
$$

we obtain the gap function $\Delta_{i j}(k)$ as an eigenstate and its eigenvalue $\lambda$, where $V(q)$ is the singlet pairing interaction as described in Eqs. (2)-(7) of Ref. [40]. The largest $\lambda$ reaches unity at $T=T_{c}$. Since $\lambda$ is monotonic and an increasing function of $T^{-1}$, we use $\lambda$ at $T=0.01 \mathrm{eV}$ as a qualitative measure of $T_{c}$ instead of calculating at $T_{c}$. In some FLEX calculations, $\lambda$ at fixed temperature is used to compare the relative height of $T_{c}$ among similar materials [69,70]. We obtain $\lambda=0.50$ for $\mathrm{La}$ and 0.71 for $\mathrm{Hg}$. This is qualitatively consistent with the experimental observation that $\mathrm{Hg}\left(T_{c}=98 \mathrm{~K}\right)$ is higher than $\mathrm{La}\left(T_{c}=39 \mathrm{~K}\right)[37,38]$.

To investigate how $U_{\mathrm{M}}$ affects $\lambda$ in more detail, we perform calculations by rescaling $U_{\mathrm{M}}$ hypothetically. We plot $\lambda$ as a function of $U^{x^{2}-y^{2}}$ in Fig. 4. In the calculation, $\widehat{H}_{\mathrm{M}}^{0}$ and the ratio between all the elements of $U_{\mathrm{M}}$ are fixed. We see that $\lambda$ increases rapidly with smaller $U^{x^{2}-y^{2}}$ and plateaus with larger $U^{x^{2}-y^{2}}$ in both materials. The cases of original $U^{x^{2}-y^{2}}$ as shown in Table II are shown by open circles. These are in the plateau region [71]. Because of the small changes in the region, $\lambda$ of the two cuprates does not change so much even if we use $U_{\mathrm{m}}$ instead of $U_{\mathrm{M}}$, where $\lambda_{\mathrm{cRPA}}^{\mathrm{La}}=0.52$ and $\lambda_{\mathrm{cRPA}}^{\mathrm{Hg}}=$ 0.64 . The difference between $\mathrm{La}$ and $\mathrm{Hg}$ is mainly from the hybridization of the $d_{x^{2}-y^{2}}$ orbital with the $d_{z^{2}}$ orbital. This is already examined by previous FLEX calculations with empirically determined interaction parameters [39]. Sakakibara et al.

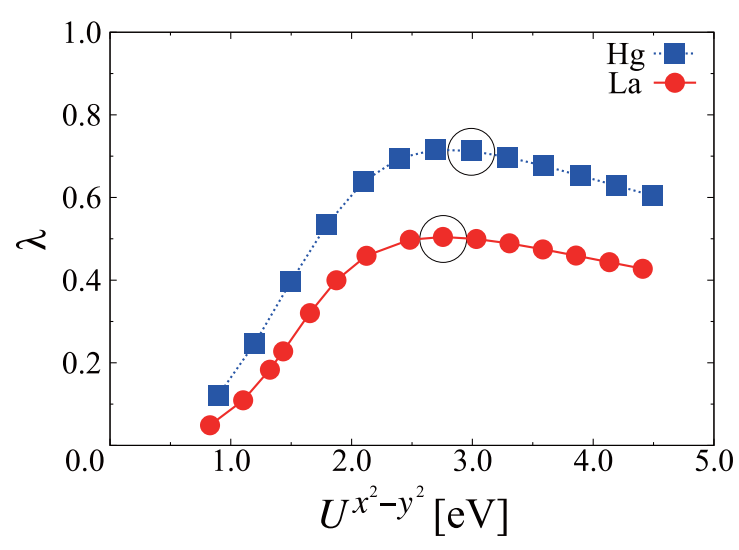

FIG. 4. The eigenvalues $\lambda$ of the Eliashberg equation are plotted as a function of $U^{x^{2}-y^{2}}$. Here the temperature is $0.01 \mathrm{eV}$. Red filled circles show the value for $\mathrm{La}$ and blue squares for $\mathrm{Hg}$. Open circles indicate the results obtained with the value shown in Table II.

already showed that FLEX reproduces the experimental trends of $T_{c}$ (see Fig. 1(a) of Ref. [42]). The detailed mechanism on how the hybridization affects $T_{c}$ was discussed in Sec. III D of Ref. [40].

\section{SUMMARY}

With mRPA we obtain the two-orbital Hubbard models for $\mathrm{La}_{2} \mathrm{CuO}_{4}$ and $\mathrm{HgBa}_{2} \mathrm{CuO}_{4}$ in first principles. The main part of mRPA is how to determine the on-site interaction parametrized by four parameters. We see that the interactions are close to those in cRPA. However, we see some differences. A difference comes from the fact that the effective size of the polarization function $P_{\mathrm{m}}$ in cRPA becomes smaller than $P_{\mathrm{M}}$ in mRPA. This is because the probability factors $c_{i}$ in Eq. (8) are distributed among the oxygen bands when $d$ - $p$ hybridization is strong, as in $\mathrm{HgBa}_{2} \mathrm{CuO}_{4}$.

For the models, we perform FLEX to evaluate superconductivity. The results are consistent with experiments. With the interaction obtained in mRPA, we confirm that $T_{c}$ is not so strongly dependent on the scale of interaction. Along the line of the combination of mRPA and FLEX, we will be able to predict new superconductors.

\section{ACKNOWLEDGMENTS}

We appreciate discussions with Dr. Friedlich, Dr. Şaşıoğlu, Dr. Imada, Dr. Arita, Dr. Hirayama, Dr. Kuroki, Dr. S. W. Jang, and Dr. M. J. Han. H.S. appreciates fruitful discussions with Dr. Misawa, Dr. Nomura, and Dr. Shinaoka. This work was supported by JSPS KAKENHI (Grants No. 16K21175 and No. 17K05499). The computing resource is supported by Computing System for Research in Kyushu University (ITO system), the supercomputer system in RIKEN (HOKUSAI), and the supercomputer system in ISSP (sekirei).
[1] C. Honerkamp, Phys. Rev. B 85, 195129 (2012).

[2] M. Kinza and C. Honerkamp, Phys. Rev. B 92, 045113 (2015)
[3] S. R. White, Phys. Rev. Lett. 69, 2863 (1992).

[4] E. Gull, A. J. Millis, A. I. Lichtenstein, A. N. Rubtsov, M. Troyer, and P. Werner, Rev. Mod. Phys. 83, 349 (2011). 
[5] D. Ceperley, G. V. Chester, and M. H. Kalos, Phys. Rev. B 16, 3081 (1977).

[6] A. Georges, G. Kotliar, W. Krauth, and M. J. Rozenberg, Rev. Mod. Phys. 68, 13 (1996).

[7] Y.M. Vilk and A.-M.S. Tremblay, J. Phys. I (France) 7, 1309 (1997).

[8] B. Kyung, J.-S. Landry, and A.-M. S. Tremblay, Phys. Rev. B 68, 174502 (2003).

[9] S. Onari and H. Kontani, Phys. Rev. Lett. 109, 137001 (2012).

[10] M. Tsuchiizu, Y. Yamakawa, S. Onari, Y. Ohno, and H. Kontani, Phys. Rev. B 91, 155103 (2015).

[11] N. E. Bickers, D. J. Scalapino, and S. R. White, Phys. Rev. Lett. 62, 961 (1989).

[12] H. Sakakibara, S. W. Jang, H. Kino, M. J. Han, K. Kuroki, and T. Kotani, J. Phys. Soc. Jpn. 86, 044714 (2017).

[13] N. Marzari and D. Vanderbilt, Phys. Rev. B 56, 12847 (1997).

[14] I. Souza, N. Marzari, and D. Vanderbilt, Phys. Rev. B 65, 035109 (2001).

[15] T. Kotani, J. Phys.: Condens. Matter 12, 2413 (2000).

[16] F. Aryasetiawan, M. Imada, A. Georges, G. Kotliar, S. Biermann, and A. I. Lichtenstein, Phys. Rev. B 70, 195104 (2004)

[17] K. Nakamura, R. Arita, and M. Imada, J. Phys. Soc. Jpn. 77, 093711 (2008).

[18] K. Nakamura, Y. Yoshimoto, T. Kosugi, R. Arita, and M. Imada, J. Phys. Soc. Jpn. 78, 083710 (2009).

[19] T. Miyake, F. Aryasetiawan, and M. Imada, Phys. Rev. B 80, 155134 (2009).

[20] T. Miyake, K. Nakamura, R. Arita, and M. Imada, J. Phys. Soc. Jpn. 79, 044705 (2010).

[21] K. Nakamura, Y. Yoshimoto, Y. Nohara, and M. Imada, J. Phys. Soc. Jpn. 79, 123708 (2010).

[22] T. O. Wehling, E. Şaşıŏlu, C. Friedrich, A. I. Lichtenstein, M. I. Katsnelson, and S. Blügel, Phys. Rev. Lett. 106, 236805 (2011).

[23] E. Şaşığlu, C. Friedrich, and S. Blügel, Phys. Rev. B 83, 121101(R) (2011).

[24] T. Misawa, K. Nakamura, and M. Imada, Phys. Rev. Lett. 108, 177007 (2012).

[25] Y. Nomura, M. Kaltak, K. Nakamura, C. Taranto, S. Sakai, A. Toschi, R. Arita, K. Held, G. Kresse, and M. Imada, Phys. Rev. B 86, 085117 (2012).

[26] K. Nakamura, Y. Yoshimoto, and M. Imada, Phys. Rev. B 86, 205117 (2012).

[27] H. Shinaoka, T. Misawa, K. Nakamura, and M. Imada, J. Phys. Soc. Jpn. 81, 034701 (2012).

[28] P. Werner, M. Casula, T. Miyake, F. Aryasetiawan, A. J. J. Millis, and S. Biermann, Nat. Phys. 8, 331 (2012).

[29] M. Casula, P. Werner, L. Vaugier, F. Aryasetiawan, T. Miyake, A. J. Millis, and S. Biermann, Phys. Rev. Lett. 109, 126408 (2012).

[30] T. Misawa and M. Imada, Nat. Commun. 5, 6738 (2014).

[31] T. Koretsune and C. Hotta, Phys. Rev. B 89, 045102 (2014).

[32] E. Şaşığlu, in Lecture Notes of the 45th IFF Spring School Computing Solids-Models, ab initio Methods and Supercomputing (Schriften des Forschungszentrums Jülich Reihe Schlüsseltechnologien, 2014).

[33] H. Shinaoka, M. Troyer, and P. Werner, Phys. Rev. B 91, 245156(R) (2015).
[34] S. W. Jang, H. Sakakibara, H. Kino, T. Kotani, K. Kuroki, and M. J. Han, Sci. Rep. 6, 33397 (2016).

[35] A. van Roekeghem, L. Vaugier, H. Jiang, and S. Biermann, Phys. Rev. B 94, 125147 (2016).

[36] M. Hirayama, Y. Yamaji, T. Misawa, and M. Imada, Phys. Rev. B 98, 134501 (2018).

[37] H. Takagi, R. J. Cava, M. Marezio, B. Batlogg, J. J. Krajewski, W. F. Peck, P. Bordet, and D. E. Cox, Phys. Rev. Lett. 68, 3777 (1992).

[38] A. Yamamoto, K. Minami, W.-Z. Hu, A. Miyakita, M. Izumi, and S. Tajima, Phys. Rev. B 65, 104505 (2002).

[39] H. Sakakibara, H. Usui, K. Kuroki, R. Arita, and H. Aoki, Phys. Rev. Lett. 105, 057003 (2010).

[40] H. Sakakibara, H. Usui, K. Kuroki, R. Arita, and H. Aoki, Phys. Rev. B 85, 064501 (2012).

[41] H. Sakakibara, K. Suzuki, H. Usui, K. Kuroki, R. Arita, D. J. Scalapino, and H. Aoki, Phys. Rev. B 86, 134520 (2012).

[42] H. Sakakibara, K. Suzuki, H. Usui, S. Miyao, I. Maruyama, K. Kusakabe, R. Arita, H. Aoki, and K. Kuroki, Phys. Rev. B 89, 224505 (2014).

[43] H. Kamimura and M. Eto, J. Phys. Soc. Jpn. 59, 3053 (1990).

[44] M. Eto and H. Kamimura, J. Phys. Soc. Jpn. 60, 2311 (1991).

[45] A. Freeman and J. Yu, Physica B+C 150, 50 (1988).

[46] X. Wang, H. T. Dang, and A. J. Millis, Phys. Rev. B 84, 014530 (2011).

[47] L. Hozoi, L. Siurakshina, P. Fulde, and J. van den Brink, Sci. Rep. 1, 65 (2011).

[48] S. Uebelacker and C. Honerkamp, Phys. Rev. B 85, 155122 (2012).

[49] L. Hozoi and M. S. Laad, Phys. Rev. Lett. 99, 256404 (2007).

[50] C. E. Matt, D. Sutter, A. M. Cook, Y. Sassa, M. Månsson, O. Tjernberg, L. Das, M. Horio, D. Destraz, C. G. Fatuzzo, K. Hauser, M. Shi, M. Kobayashi, V. N. Strocov, T. Schmitt, P. Dudin, M. Hoesch, S. Pyon, T. Takayama, H. Takagi, O. J. Lipscombe, S. M. Hayden, T. Kurosawa, N. Momono, M. Oda, T. Neupert, and J. Chang, Nat. Commun. 9, 972 (2018).

[51] J. D. Jorgensen, H. B. Schüttler, D. G. Hinks, D. W. Capone II, K. Zhang, M. B. Brodsky, and D. J. Scalapino, Phys. Rev. Lett. 58, 1024 (1987).

[52] J. Wagner, P. Radaelli, D. Hinks, J. Jorgensen, J. Mitchell, B. Dabrowski, G. Knapp, and M. Beno, Physica C: Superconductivity 210, 447 (1993).

[53] A first-principles electronic-structure suite based on the PMT method, ecalj package, is freely available from https://github. com/tkotani/ecalj. Its one-body part is developed based on the LMTO part in the LMsuit package at http://www.lmsuite.org/.

[54] T. Kotani, M. van Schilfgaarde, and S. V. Faleev, Phys. Rev. B 76, 165106 (2007).

[55] T. Kotani, J. Phys. Soc. Jpn. 83, 094711 (2014).

[56] D. Deguchi, K. Sato, H. Kino, and T. Kotani, Jpn. J. Appl. Phys. 55, 051201 (2016).

[57] In our previous paper [12] we made a wrong statement that $U_{\mathrm{M}}$ would become smaller if we consider off-site interactions.

[58] We use the tetrahedron method [54,55] in the Brillouin zone to calculate the matrix $W$ and $U_{\mathrm{m}}$, where we use $8 \times 8 \times 8(8 \times$ $8 \times 4) k$ points for $\mathrm{La}_{2} \mathrm{CuO}_{4}\left(\mathrm{HgBa}_{2} \mathrm{CuO}_{4}\right)$. For a model calculation to determine $U_{\mathrm{M}}^{122^{\prime} 1^{\prime}}$, we take $64 \times 64 \times 4 k$-point grids 
for discrete summation. We use dense enough 4096 Matsubara meshes at $T=0.005 \mathrm{eV}$ (virtually equal to $T=0 \mathrm{eV}$ ).

[59] Y. Ohta, T. Tohyama, and S. Maekawa, Phys. Rev. B 43, 2968 (1991).

[60] O. Andersen, A. Liechtenstein, O. Jepsen, and F. Paulsen, J. Phys. Chem. Solids 56, 1573 (1995), proceedings of the Conference on Spectroscopies in Novel Superconductors.

[61] E. Pavarini, I. Dasgupta, T. Saha-Dasgupta, O. Jepsen, and O. K. Andersen, Phys. Rev. Lett. 87, 047003 (2001).

[62] M. Mori, G. Khaliullin, T. Tohyama, and S. Maekawa, Phys. Rev. Lett. 101, 247003 (2008).

[63] C. Weber, K. Haule, and G. Kotliar, Phys. Rev. B 82, 125107 (2010).

[64] C. Weber, C. Yee, K. Haule, and G. Kotliar, Europhys. Lett. 100, 37001 (2012).
[65] A. I. Lichtenstein and M. I. Katsnelson, Phys. Rev. B 57, 6884 (1998).

[66] K. Yada and H. Kontani, J. Phys. Soc. Jpn. 74, 2161 (2005).

[67] M. Mochizuki, Y. Yanase, and M. Ogata, Phys. Rev. Lett. 94, 147005 (2005).

[68] T. Takimoto, T. Hotta, and K. Ueda, Phys. Rev. B 69, 104504 (2004).

[69] H. Ikeda, R. Arita, and J. Kuneš, Phys. Rev. B 81, 054502 (2010).

[70] K. Kuroki, H. Usui, S. Onari, R. Arita, and H. Aoki, Phys. Rev. B 79, 224511 (2009).

[71] The correlation between $U / t$ and $T_{c}$ is discussed with Hubbard model calculations, e.g., in Ref. [72].

[72] H. Yokoyama, M. Ogata, Y. Tanaka, K. Kobayashi, and H. Tsuchiura, J. Phys. Soc. Jpn. 82, 014707 (2013). 